\title{
EFFECTS OF A NIGHT VISION ENHANCEMENT SYSTEM (NVES) ON DRIVING: RESULTS FROM A SIMULATOR STUDY
}

\author{
Erik Hollnagel \\ CSELAB, Department of Computer and Information Science \\ University of Linköping, SE-58183 Linköping, Sweden \\ E-mail: erik.hollnagel@ida.liu.se \\ Jan-Erik Källhammer \\ Autoliv Research \\ 44783 Vårgårda, Sweden \\ E-mail: jan-erik.kallhammer@autoliv.com
}

\begin{abstract}
Summary: Three related experiments looked at the effects of an NVES on driving performance, with differences in image size ratio, lateral position and direct/indirect viewing as parameters. The experiments used experienced drivers in a fixed based virtual-reality driving simulator. Experiment 1 found that subjects using an NVES gained time to assess the situation and choose an appropriate response, which was seen in terms of better control of braking and swerving. Contrary to expectations, subjects did not drive significantly faster when using the NVES. Experiment 2 found that a 1:2 display ratio resulted in better anticipatory control without any adverse effects from differences in recognition distances. When using an NVES display displaced laterally from the normal line of sight, drivers kept the vehicle closer to the middle of the road. They also found the displaced position less favourable than one in the normal line of sight, although there were no strong negative effects of a the displacement. Experiment 3 compared a virtual (collimated) display to a direct viewing Flat Panel, with the hypothesis that reduced need of accommodation would lead to smoother driving. The results showed some differences between the two display types, although they were small compared to the effects of learning. Altogether the experiments confirmed that an NVES leads to an indisputable improvement in the drivers' anticipatory control, and hence has considerable safety potential. This work has also emphasised the need to consider the combined effects of an NVES as a system on driving, rather than to do classical controlled experiments.
\end{abstract}

\section{INTRODUCTION}

The ability to drive efficiently and safely requires both closed-loop (feedback) and open-loop (feedforward) control (McRuer et al., 1977). Drivers do not only react or respond to what exists here and now, but also try to anticipate what may come ahead. Effective anticipation prepares the driver for what may happen and thereby reduces overall workload. The basis for anticipation is the ability to combine what can be seen with knowledge about environment topography and dynamic traffic characteristics. Experienced drivers generally look quite far ahead, approximately 100-400 meters depending on road conditions and the speed of the vehicle (Rumar, 1991). Since the driver's visual range is seriously reduced at night, typically to about 80 metres, the ability to anticipate is diminished and the overall level of control consequently 
suffers, with driving reactions becoming more sudden and less accurate. The purpose of a Night Vision Enhancement System (NVES) is partly to compensate for this reduction by enabling the driver to detect objects too faint to be seen by the naked eye. This is done by converting images from an infrared (IR) camera and showing them on a monochrome display somewhere in the car.

\section{GENERAL AND SPECIFIC RESEARCH QUESTIONS}

Two main questions relating to the use of an NVES are (1) whether it provides any benefit to the driver, and (2) whether it introduces any risks. The research that has been done so far supports the conclusion that NVESs have considerable safety potential because they increase drivers' visual range when it is dark. As far as the possible risks go, there is only indirect evidence (e.g., Gish, 2001), although it is prudent to assume that an NVES carries with it some risks, as any new kind of technology does. The most tangible risk derives from the fact that using an NVES requires some of the driver's attention, which affects his or her ability to focus on the primary driving task.

On a more detailed level, a large number of aspects relating to the use of an NVES may be raised (cf. Rumar, 2002), such as position of the image in X, Y, and Z dimensions; image size (ratio between the retinal sizes of objects in the image and objects in the real world); image proportions; image's visual qualities (brightness, brightness contrast, resolution); and whether the image is transparent or opaque. A change to any of these aspects will have effects on driving performance (Smiley, 2000). In some cases these effects can be inferred directly from established theories about human cognition and performance, but in most cases neither the empirical nor the theoretical basis allow definite conclusions to be made. Furthermore, many aspects affect each other, such as image size and image proportions, or transparency and brightness. It is therefore more important to consider the combined effects on driving of an NVES as a system than to experiment with each aspect as an independent variable.

\section{APPARATUS AND METHOD}

Various configurations of a simulated NVES were tested in a fixed-base simulator at the VR laboratory at Linköping University (Figure 1). The NVES system emulated passive (far) IR camera technology, where camera optical field of view, camera image characteristics and display types could be varied. The display was either a virtual image where the apparent distance was further away than the physical distance (Indirect View Panel or IVP) or a directly viewed Flat Panel display (Direct View Panel or DVP). The simulator software included a full vehicle model and road system. The physical environment comprised a front seat, main instrument panel and steering wheel from a Saab 9-5, brake and accelerator pedals, but no mirrors or any other equipment. The simulator offered a $115^{\circ}$ degree Field of View (FoV) on a custom-built projection wall via three Sony LCD video projectors.

A 95.8-kilometer road was modelled for the study, divided into two main sections of approximately equal length. In each section,

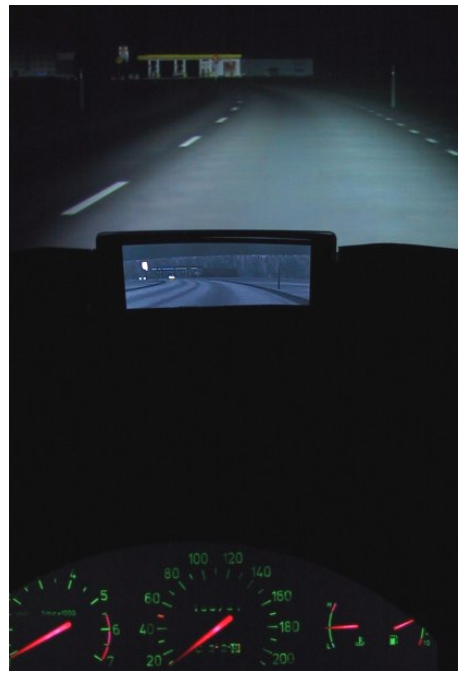

Figure 1. Simulator with IVP NVES display 
drivers encountered a few potentially hazardous objects (moose, deer, parked cars, people) and also met other vehicles on the road. The environment was a rural Swedish landscape and changed between forests and fields in order to make the driving experience as real as possible. There were traffic signs but no road crossings. For all experiments, the subjects were experienced drivers from local high-tech industries who had responded to an announcement. Because of self-selection, most subjects were male. They had all held a driver's license for more than five years and driven a minimum of 10,000 kilometres per year. Experiments usually took place during the late afternoon or early evening. All experiments began with a test drive $(\sim 15$ minutes) without an NVES, so that subjects could get used to the simulator and practice night driving. This was followed by two experiment sessions where conditions varied depending on the NVES configuration being tested. The sessions usually took about 45 minutes each to complete, depending on speed. The subjects thus drove for nearly two hours in simulated night conditions, with short breaks in between sessions.

\section{EXPERIMENT 1}

This experiment investigated the impact of using a NVES, and also looked at the effects of image size and brightness contrast (Hollnagel et al., 2001; Karlsson, 2002). It used 40 male subjects in a between-groups design, where subjects drove in simulated night conditions for about 40 kilometres without and 80 kilometres with an IVP NVES display. (The road was segmented differently than in the following experiments.) As shown by Figure 2, subjects with the NVES detected objects from a distance of around 400-500 meters, and had more control of braking and swerving than in the without-NVES condition. Subjects clearly used the additional time to assess the situation and choose an appropriate response. Contrary to expectations, there were no significant differences in speed between the two conditions. A questionnaire-based debriefing found that almost all subjects appreciated the improved visual information, although some commented that the NVES required too much attention and that it was difficult to judge the position of objects on the road.

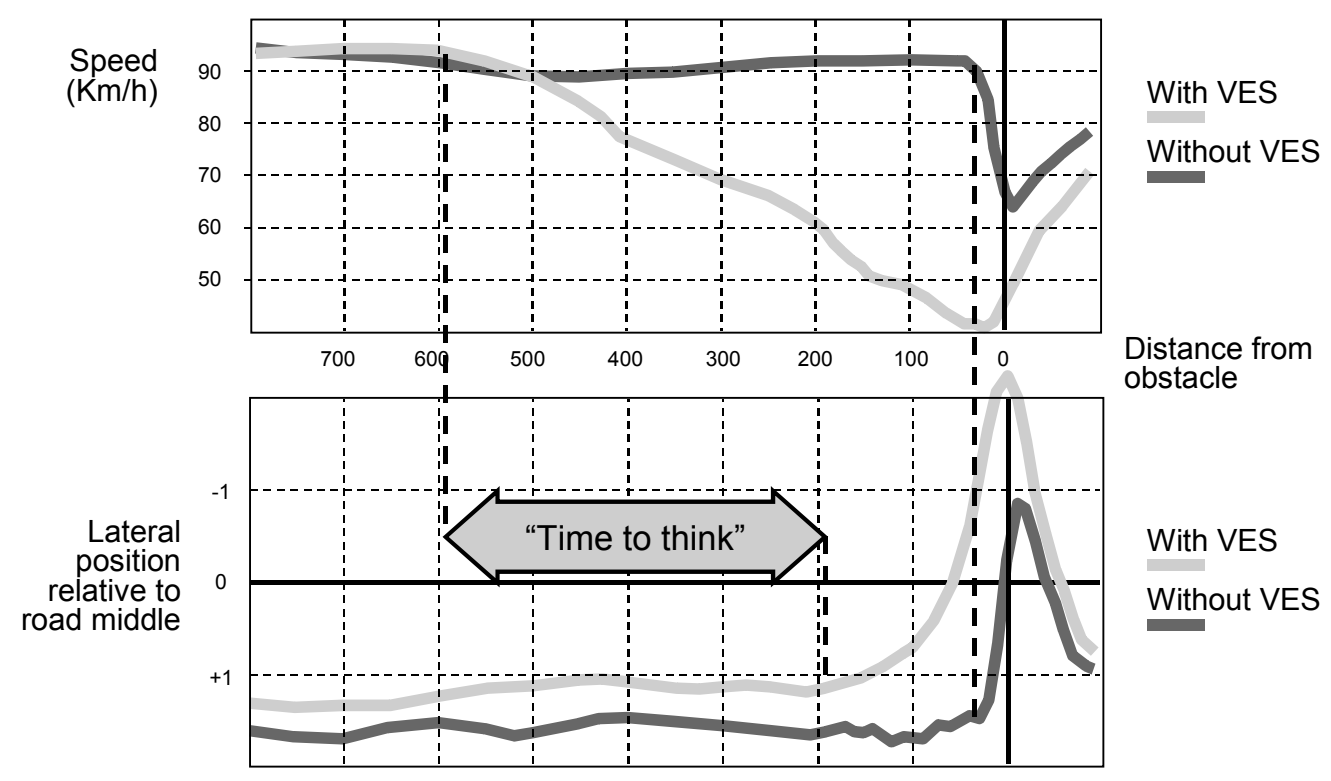

Figure 2. Average avoidance manoeuvres for moose standing on the road in driver's lane 


\section{EXPERIMENT 2}

This experiment looked at several aspects of NVES usage, namely different image size ratios and different lateral positions of the display. It also considered the potential for cognitive capture from a bright NVES display. The experiment used the same facility as in the first study, although the road configuration was redesigned to make comparison of performance from different conditions easier. The experiment used a within-groups design, with $2 \times 8$ subjects for each of the three main conditions (image ratio, position, brightness), for a total of 48 subjects (male and female). After the practice session, each group drove two different but comparable routes with an NVES display present in one of the six configurations (two image size ratios, two lateral positions, two brightness conditions).

Different image size ratios were investigated using a non-transparent IVP image overlay on the projection wall in front of the driver, approximately $2^{\circ}$ under the eye ellipse. The IVP extended $12^{\circ}$ horizontally in the FoV and $4^{\circ}$ vertically. The (simulated) camera had an opening angle of either $12^{\circ}$ or $24^{\circ}$, thereby producing images with either $1: 1$ or $1: 2$ display size ratios. Since a $24^{\circ}$ camera angle effectively doubles the horizontal FoV, the driver can see more of the road and is therefore able better to anticipate what might happen both for straight roads and driving through curves. At a speed of $100 \mathrm{~km} / \mathrm{h}$, the critical distance to detect an obstacle in order to stop in time is about 70 meters. When using an NVES with a $12^{\circ}$ camera angle (1:1 display ratio), the driver can see seven meters to either side of the road 70 meters ahead, and 42 meters to either side 400 meters ahead. For a $24^{\circ}$ camera angle (1:2 display ratio), the corresponding values are about 15 and 85 meters, respectively. A 1:2 display ratio resulted in better anticipatory control without any adverse effects from possible differences in recognition distances (Grönqvist, 2002).

The effects of different lateral positions was investigated using a DVP with a 1:2 display ratio either in the driver's line of sight when looking straight ahead or displaced towards the middle of the car, about $24^{\circ}$ to the right. In order see the displaced display, the driver had to take his/her eyes off the road. It was expected that this would affect the quality of driving, seen via the measured performance indicators (braking profile and lateral variability). The need to look more closely at an object visible in the DVP image would further detract from the primary driving task, and thereby amplify the effects of the lateral displacement. An ANOVA for standard deviations of speed showed a small but significant difference between the two display positions $(p=0.047)$, where the display in front of the driver resulted in smoother driving. There was also a significant difference in lateral road position between display conditions, so that drivers with the displaced display drove closer to the middle of the road. According to the model (Hollnagel, 2002), driving closer to the middle of the road buys the driver more time to respond if anything unexpected appears. (Note that this strategy obviously only works if there is little other traffic.) There were no significant differences in the swerving response to objects in the scenarios. It was also found that drivers were generally in favour of a display in the normal line of sight (Druid, 2002).

The effect of cognitive capture was investigated using an IVP display on either a transparent or a non-transparent projection surface (Nilsson, 2003). As the IVP on the transparent surface had to be brighter in order for the image to be properly seen against the varying backgrounds, there was a risk of cognitive capture. The luminance ratios between the two conditions were about 1:2.5. 
No significant capture effects were found, although this was probably due to inappropriate experimental conditions (test stimuli).

\section{EXPERIMENT 3}

This experiment looked at the effects of using DVP and IVP (collimated) displays. The DVP display was a TFT flat screen monitor of which only a portion was used for the NVES image, the unused area being masked with black cardboard. The viewable area had an aspect ratio of approximately 1:3, corresponding to an image of 120x320 pixels. The IVP was custom built and corresponded in size and resolution to the DVP, with the virtual image appearing approximately $250 \mathrm{~cm}$ in front of the driver. It was expected that the IVP-display would reduce the need for reaccommodation and hence save time when switching attention between display and environment, and that the effect of this would be seen in a smoother deceleration. Sixteen male participants drove $2 \times 45$ kilometres in a one-way repeated-measures design, counterbalancing the order of presentation of the displays.

Small but significant differences were found in magnitude of deceleration, but not in beginning point of deceleration, point of applying brakes, or point of maximum deceleration. It was concluded that while there may be differences between displays, these are probably smaller than the difference between driving with and without an NVES. Questionnaire replies showed that drivers found it easier to detect objects in the IVP than in the DVP, although this could possibly be an effect of slightly different contrast calibration of the displays. Questionnaire replies also indicated that drivers believed they used the IVP more than the DVP.

\section{CONCLUSIONS}

It must be remembered that a driving simulator is no more than a substitute for studies under real-life conditions. The simulator used in these experiments was fixed-base, but had excellent visual and auditory qualities. Many effects of NVES usage can only be studied realistically during actual driving, although this will require considerable cost and effort. A simulator is a powerful and convenient tool that can resolve some basic issues and be used to improve the focus of field studies, but it cannot in itself provide full answers. Given these limitations, the results confirm the findings from previous experiments that NVESs produce an indisputable improvement in drivers' anticipatory control, and hence have considerable safety potential. The earlier detection of objects made possible by an NVES buys the driver valuable time to assess the situation and prepare a measured response. There also seems to be an advantage to having a wider field of view and to placing the NVES in the normal line of sight of the driver, although there were no strong negative effects of a lateral displacement.

The consensus in the driving research community, as summarised by Rumar (2002), is that in night driving visual guidance is less impaired than target detection. An NVES should therefore help with the detection of objects rather than with the recognition of objects. Indeed, in driving - either during day or night - the first priority is to know something is on the road ahead, while the second is to know what it is. This clearly has consequences for how the information should best be presented. 


\section{REFERENCES}

Druid, A. (2002). Vision enhancement systems - Does display position matter? Masters Thesis, Department of Computer and Information Science, Linköping University, Sweden.

Gish, K. V. (2001). Driver behavior and performance using an infrared night vision enhancement system. Washington, D.C.: Office of Crash Avoidance Research, National Highway Traffic Safety Administration (Grant No. DTNH22-95-D-07019).

Grönqvist, H. (2002). Visual enhancement systems - The importance of field of view. Masters Thesis, Department of Computer and Information Science, Linköping University, Sweden.

Hollnagel, E. (2002). Time and time again. Theoretical Issues in Ergonomic Sciences, 3(2), 143158.

Hollnagel, E., Karlsson, J., Magnusson, T. \& Taube, U. (2001). They drive at night - can visual enhancement systems keep the driver in control. Proceedings of Driving Assessment 2001, Snowmass, CO., August 14-17, 2001

Karlsson, J. (2002). Vision enhancement systems - Support for night-time driving? Masters Thesis, Department of Computer and Information Science, Linköping University, Sweden.

McRuer, D. T., Allen, R. W., Weir, D. H., \& Klein, R. H. (1977). New results in driver steering control. Human Factors, 19, 381-397.

Nilsson, J. (2003). A comparison between opaque and transparent displays for vision enhancement systems. Masters Thesis, Department of Computer and Information Science, Linköping University, Sweden.

Rumar, K. (1991). Ögat och mörkertrafiken. Skandia, Team Tryck AB, Stockholm.

Rumar, K. (2002). Night vision enhancement systems: What should they do and what more do we need to know? (Report No. UMTRI-2002-12.) Ann Arbor, Michigan: The University of Michigan Transportation Research Institute.

Smiley, A. (2000). Behavioral adaptation, safety, and intelligent transportation systems. Transportation Research Record 1724, Paper No. 00-1504. 